\title{
Wireless Condition Monitoring Technique of Power Transformer and Formulation of Health Index
}

\author{
Bhoopesh Nemichand Chaudhari ${ }^{1}$, Makarand Sudhakar Ballal ${ }^{2}$ \\ ${ }^{1}$ Associate Professor, Department of Electrical Engineering, P E S Collage of Engineering, Aurangabad, India \\ ${ }^{2}$ Associate Professor, Department of Electrical Engineering, Vishvesshra National Institute of Technology, Nagpur, India
}

\begin{abstract}
In this paper remote condition monitoring system is developed using single board computer and data acquisition system which senses different parameters and communicated to the remote end using internet. The economical aspect of transformer with remote condition monitoring system which gives necessary information of operating condition is analyzed. The performance of remote condition monitoring system is tested on 16 KVA transformer in the laboratory. Transformers with remote condition monitoring system can be provided maintenance as and when required.
\end{abstract}

Keywords: Remote condition monitoring system (RCMS)

\section{Introduction}

Transformer is a static piece of equipment with a complicated electromagnetic circuit inside. Although the transformer is a complex piece of equipment, proper designing of an insulation system, magnetic circuit, structure and selection of proper transformer auxiliaries make transformer extremely reliable equipment. Utilities are very much keen to control and monitor the status and condition of all transformers fleet so they can intervene before a failure or malfunction of it. Many of the transformers in use at utilities have already exceeded design life. Most power transformer will encounter emergency overloads on occasions, with subsequent loss of life. The load peaks may be predictable or non predictable, unexpected generates high temperatures which shortens transformer lifetime. Presently the load and the age of the apparatus is increasing and therefore the monitoring and diagnosis of power transformers becomes more and more important, whereby monitoring is the collection of relevant data during service (online) or during maintenance or test periods (offline) and diagnostic is the technical evaluation and interpretation of the recorded data. Monitoring improves the reliability of the assets by continuously keeping a watchful eye on the most critical transformer components. Inordinate temperature rise in a power transformer due to load current have known to be the most important factor in causing rapid degradation of its insulation and decides the optimum load carrying ability or the load ability at a transformer.

In [1] hourly load and ambient temperatures obtained through condition monitoring are used to assess the operating profile of the equipment. The only inputs to the IEEE life consumption models to assess the consumed life of insulation estimated are restricted to load factors and ambient temperatures. However, this approach needs to be extended to include the long-term shift effects in load and ambient temperature variations. A laboratory controlled thermal aging experimental method has been described in [2] to obtain information on the status of insulation by diagnostic testing and condition monitoring has been suggested. However, to correct the results to field conditions, a correction factor is required for certain diagnostic parameters. The monitoring methods presented in [3] are based on the existing IEC and IEEE standards and neural-network analysis. These methods are used in to calculate the top-oil and hot-spot temperature as well as the loss of life of a transformer. This requires measured data from the studied or same type of transformer supplying similar loading.

Article [4], classified the defect sources using phase-resolved partial discharge patterns. But, this requires an understanding of the traits associated with, and relationship between, observed partial discharge activity and responsible defect sources. The application of Fiber Bragg Grating (FBG) sensors for monitoring power transformer hot-spot temperatures has been demonstrated in [5]. However, the temperature sensors are based on proprietary encapsulated FBG sensors and the optical interrogation unit requires a special designed narrowband high power broadband fiber conducted in this work, a further investigation needs to be undertaken to verify source. Model-based identification approach has been developed in [6] to determine parameters of a demagnetized transformer core such as magnetic permeability and electrical conductivity, of power transformers on the basis of frequency response analysis (FRA). However, only simulation studies are the proposed identification approach with experimental measurements. In [7] an evidential reasoning ER-based approach to transformer winding condition assessment using FRA is proposed to formalize a frequency response analysis (FRA) evaluation process for the first time. In this study, only the experimental FRA data from a normal transformer is used as a winding condition evaluation. Much research needs to be carried out to establish more reliable interpretation features with regard to different FRA diagnoses using experimental FRA data related to various winding failure conditions from real transformers. The technique of Conditional Anomaly Detection and its application to transformer monitoring described in [8]. However, various system components are needed to provide a solution that supports engineers in decision 


\section{International Journal of Science and Research (IJSR) \\ ISSN (Online): 2319-7064 \\ Index Copernicus Value (2013): 6.14 | Impact Factor (2014): 5.611}

making. The main components of power transformer are the windings, bushings, transformer oil, core, tank, cooling system, and tap changer.

Failure analysis of large power transformers can be beneficial in determining which component is more important in evaluating the condition of transformers. Statistics [9] show that the most frequent causes followed by long outage damages are in tap changer, active component and in bushings, as shown in table I. Power organizations today use either or both of the following traditional maintenance models. On the one hand, "failure-based" or "reactive" maintenance occurs when equipment is left un-monitored until one or more failure modes have been observed. On the other hand, "preventive" maintenance approach involves physically visiting an asset on a regularly scheduled basis, assessing the performance of that asset and then applying necessary maintenance to ensure optimal performance.

On the bases of CIGRE report, the failure pattern of power transformers follows a "bath-tub" curve for 400 transformers, as shown in Fig. 1 [10]. The first part of the curve is failure due to infant mortality; the second part of the curve is the constant failure rate; and the last part of the curve is failure due to aging. The numbers of transformer failures in the second part is greater than the last part. Electrical stresses, Electromagnetic stresses, Dielectric stresses, Thermal stresses, and Chemical stresses can cause premature failure. The accuracy of the predictions is not always as good as are desired. Unacceptable temperature rise may occur due to several fault conditions other than overloading, and hence the need of an online monitoring of the transformer becomes more prominent.

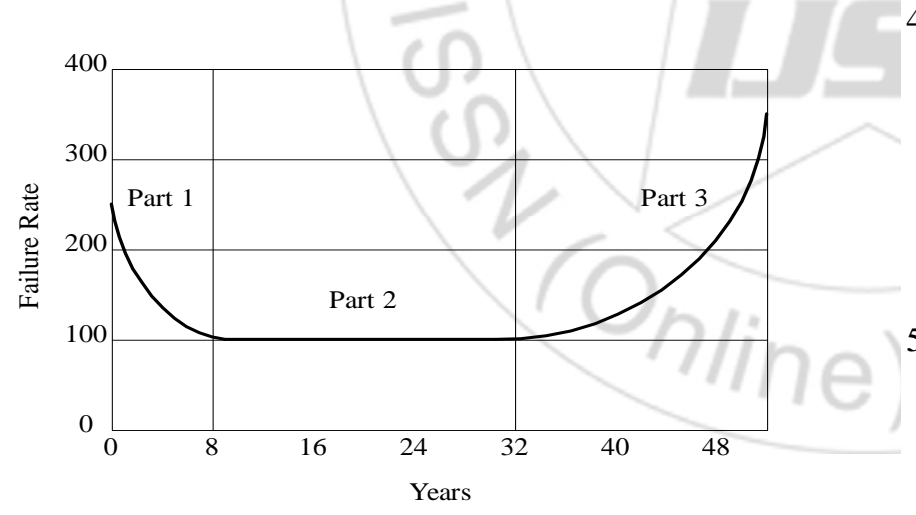

Figure 1: Transformer Failure Bath tub curve

The accuracy of the predictions is not always as good as are desired. Unacceptable temperature rise may occur due to several fault conditions other than overloading, and hence the need of an online monitoring of the transformer becomes more prominent.

\section{Condition Monitoring}

Condition Monitoring Techniques are broadly classified as conventional and non conventional. Thermography, Dissolved Gas Analysis, Furan Analysis and Tan Delta tests are conventional where as Sweep Frequency Response analysis SFRA, Partial Discharge Measurement PD, Recovery Voltage Measurement RVM, Size exclusion Chromatography SEC, Expert System are the Non Conventional Condition Monitoring Techniques. As per the literature available Different Methods Used for Condition Monitoring of Transformer are studied .Different Types of Condition Monitoring techniques with procedure and type are as follows.

1) IR measurement: Application of DC voltage from the instrument (Megger) and comparative study of IR values at different time (15 sec60sec, $600 \mathrm{sec}$ ) gives rise the idea of the insulation. It gives Condition of the insulation (Oil and solid) Procedure is OFF line and Indecisive for fault location but provides condition assessment Routine/ investigation Type.

2) Capacitance and $\tan \delta$ measurement :By the use of Schering bridge technique and other available methods, the capacitance of dielectric materials and associated factors (Dissipation factor, Tan $\delta$ factor) are calculated. It gives Condition of the insulation (Oil and solid) Procedure is OFF line / On line and Indecisive for fault location but provides condition assessment. Routine/ investigation Type.

3) Low voltage injection tests (Voltage Ratio, Voltage Balance, Excite current, SC current etc) : Supply of Low voltage to one side of transformer and measurement of different electrical parameters on open condition and short condition, gives rise the idea for diagnosis. It gives overall conditions of Transformer core and winding. Procedure is Offline and is partially decisive for fault location. Routine / Fundamental Investigation type.

4) Dissolve Gas Analysis: Because of the insulation decomposition, different characteristic gasses evolve and dissolve in oil. The concentrations of these gases are regarded as the primary diagnosis for obtaining the condition of the insulation as well as the diagnosis of fault. It gives Condition of insulation and Primary diagnosis of fault. Procedure is Offline and gives Partial decisive for fault location. It is a Routine/ Investigation type.

5) Laboratory Testing of In-service Oil (Water content, Acidity, Inhabitation content, Interfacial tension, flash point, Pour Point, Viscosity, Dielectric strength, sp. resistance According to the IS Methods, Oil tests are conducted to obtain different electrical, physical and chemical properties for comparison of the same with the standard values. It gives condition of Transformer oil. Procedure is Off line and Indecisive for fault location but primary detection of faulty portion. It is a Routine type.

6) Turns Ratio Test: By the use of reference transformer, the testing transformer turns are compared. The ratio meter has null detector. According to the voltage excitation and current through the windings of the transformers, adjustments are tried to obtain null deflection for turn's ratio. Digital ratio meters are also available for finding the ratio. It gives Condition of windings and Condition of Tap Positions. Procedure is Off line and Indecisive for fault location but primary detection of faulty portion. It is a Routine/Primary Investigation type.

Volume 5 Issue 3, March 2016 


\section{International Journal of Science and Research (IJSR) \\ ISSN (Online): 2319-7064 \\ Index Copernicus Value (2013): 6.14 | Impact Factor (2014): 5.611}

7) Winding Resistance Measurement: Basic method is voltmeter ammeter principle. Some cases Kelvin's double bridge principle is also used for finding the DC resistance of the winding. It gives Condition of windings 2.Condition of Tap Positions.

8) Partial discharge measurement: 1. Electrical detection method - By the application of Power frequency voltage, called PDIV (Partial Discharge Inception Voltage), certain charge is allowed to develop, across the insulations. Then the developed charge is discharged by decreasing the voltage called PDEV (Partial Discharge Extinction Voltage). According to the pattern of PDIV and PDEV; the status of insulation is determined.2. Acoustic Method Because of the fault in the transformer, the solid insulation parts being heated produce acoustic waves. By this method the sensors that fixed on the inner wall of the transformer, receive the signals for analysis of the fault by computing machines. It gives Condition of Insulation (Solid).Off line procedure, Acoustic method is decisive for fault location It is a Investigation type.

9) Polarization spectrum technique / Recovery voltage measurement (RVM): It is similar to PD Electrical detection method. But charging and discharging of the insulations are done for a certain pre-determined time. According to the recovery voltage that obtained during discharge condition, the status of insulation is determined. Moisture Trend analysis of winding and paper insulation. It is Expensive and Offline, Indecisive for fault location but primary detection of faulty portion and Investigation type.

10) FRA (frequency Response Analysis) : By the use of standard spectrum analyzer, sweep frequency sinusoidal source of approx. 2V RMS is applied across the winding terminals. For different range of frequency, the impedance is measured. The curve (Impedances versus frequency) becomes indicative to know the status of the winding. The other curves for voltage ratio versus frequency are also used. It gives Mechanical displacement of winding and Dynamic response of winding. is a Expensive Offline and disconnection required .It is Partial decisive for fault location Investigation type.

11) DP measurement: By this method DP (Degree of Polymerization) value is counted for determination of the status of solid insulation and accordingly the assessment of residual life of transformer. D.P. Value: - It is average number of glucose rings in cellulose molecule. D.P. value for new paper 1000-1400 It gives Condition of Solid Insulation. And Residual life assessment. Expensive and Offline routine type. Indecisive for fault location but only assess the condition of the insulation.

12) ARY-MAP Analysis: The graphic representation of the amount of $(\mathrm{CO}+\mathrm{CO} 2)$ gas versus the service period transformer represents the degree of ageing of insulation. It gives Condition of Solid Insulation, Residual life assessment. It is Expensive, Routine type Offline and Indecisive for fault location.

13) FFA (Furfuraldehyde Analysis): By HPLC (High Performance Liquid Chromatography), furan compounds are detected and according to the concentration and rate of generation of 2-furfural, the assessing of condition and remaining life of paper of insulation are obtained. It gives Condition of Solid Insulation, Residual life assessment. Procedure is Easy and Simple, Off line and Indecisive for fault location but only assess the condition of the insulation. It is a Routine type.

Transformer condition monitoring techniques available in literature are mainly used for power transformers. All monitoring techniques are applied periodically on oil power transformer for full assessment. The result if these procedures are used to evaluate the most cost effective option when transformer is in service. or under maintenance or under repair. It helps to take replacement decision also. There is a need of developing a remote condition monitoring system incorporating more parameters like temperature, oil level, humming noise etc. Advancements in information technology and communication can aid such a system effectively.

\section{Proposed Model: Introduction}

It is evident that an RCMS for transformer is essential. A proposed model for remote condition monitoring of transformer is presented here. It includes both hardware and software implementation. The communication based on GSM or GPRS is not taking the data continuously, which again is a drawback. In the new model proposed different parameters like temperature and oil level that directly acts as a health monitor are incorporated. The monitoring system is explained by going through different aspects like data acquisition, processing, communication and the applications.

\section{A) System Architecture}

The proposed model is explained using the functional diagram as shown in Fig2. It consists of data acquisition, power supply for different components, processing, communication and a Human Machine Interface (HMI). Different parameters are sensed using sensors and passed to processing unit, which is Single Board Computer (SBC) Friendly ARM Mini2440. Then it is communicated to the remote end where decision needed to be taken regarding maintenance and protection. Computational Algorithm is developed and implemented in RCMS.

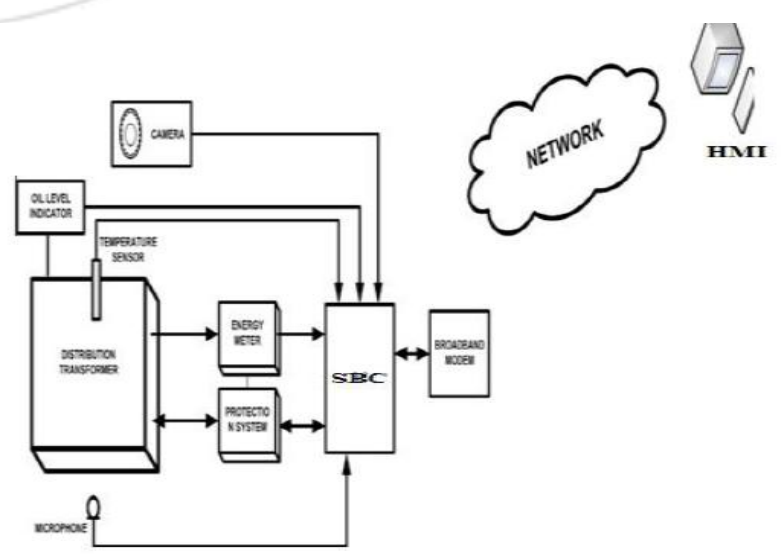

Figure 2: Functional Block Diagram 


\section{International Journal of Science and Research (IJSR) \\ ISSN (Online): 2319-7064 \\ Index Copernicus Value (2013): 6.14 | Impact Factor (2014): 5.611}

\section{B) Algorithm}

Computational Algorithm is developed which includes following steps.

1) Number of parameters available at the user end is checked. HI calculation accuracy depends on available parameters.

2) Five parameters are monitored using RCMS. Among these four parameters, which are temperature, oil level, loading and humming noise can be directly used. Image needs proper processing. Nameplate details and test results of a transformer gives the rated values as well as allowable maximum limits of these parameters. With respect to its rated values, all parameters are normalized.

3) The value of each parameter is used to calculate the Health Index Factor (HIF) which varies between 0 to 4 . Zero corresponds to the most severe condition and 4 corresponds to healthy state. Range of parameters for making this discretion have to be made after testing.

4) Each parameter is given weight age according to their effect on overall HI. This value varies between 10 to 1 in this algorithm.

5) Then the following equation is used to calculate $\mathrm{HI}$ of transformer

$$
\mathrm{HI}=\frac{\sum_{j=1}^{n} k_{f} H I f_{J}}{\sum_{j=1}^{N} 4 k_{I}} \times 100(1)
$$

6) The value of HI can be used to identify health status of transformer and is shown in application.

\section{C) Experimental Setup}

A transformer is constructed for testing this system. Special arrangements have been made for incorporating all sensors and to change the internal conditions. The parameters are varied to test RCMS. All electrical parameters can be sensed from energy meter data read out which is a serial port using RS232. Voltages, currents, power, power factor and other tampering data are available in energy meter system memory. An L \& T energy meter is used for this purpose. It is interfaced with SBC and then communicated to the remote end. Temperature of oil in the top of the tank is sensed with a thermistor type sensor (LM3). The output of the sensor that will be a voltage in accordance with temperature can be interfaced with SBC after passing through ADC. Temperature as well as oil level is interfaced with SBC using the PIC16F877A microcontroller. Its ADC's are used for converting the analog signal output of sensors to digital values. Then serial communication using RS232 passes this data to SBC When float moves, resistance varies and thereby PIC ADC senses the variation in output voltage. Interfacing with $\mathrm{SBC}$ is same as shown for temperature sensor. Both temperature and oil level are sent using same serial port from PIC. The application software developed consists of two windows. In first window, parameter values are shown and in second window, analysis is done. Health index algorithm is written in MATLAB but the results are shown in first window. Humming noise analysis consists of frequency spectrum of noise. This MATLAB figure will be shown on the application window.

\section{Experimental Result}

Detailed test results prove that RCMS can be used for transformers. Different parameter variations are discussed and corresponding $\mathrm{HI}$ is also calculated using program written in MATLAB. So it is evident that this system is efficient in determining the health status of Transformer. Operator will be able to make a decision without inspecting the transformer on site.

Table 1: Test Results

\begin{tabular}{|c|c|c|c|c|}
\hline Sr. No & Temperature & Oil Level & Loading & Health status \\
\hline 1 & Low & High & $70 \%$ & Good \\
\hline 2 & High & High & $110 \%$ & Alarm state \\
\hline 3 & Low & $90 \%$ & $70 \%$ & Good \\
\hline 4 & High & $70 \%$ & $70 \%$ & Alarm state \\
\hline 5 & High & $70 \%$ & $100 \%$ & Maintenance is required \\
\hline
\end{tabular}

\section{Conclusion}

A remote condition monitoring system is developed and tested. The analytical method used is based on health index, which is found to be effective for transformers.

Condition based maintenance and control of transformers is achieved through this work. This new system is monitoring other internal parameters also which directly influence the internal condition of the transformer and thereby ageing. RCMS implementation can reduce failure and ageing rate of transformers. It is a completely online system where instantaneous parameter variations are monitored.

\section{References}

[1] Kshira T. Muthanna, Abhinanda Sarkar, Kaushik Das, and Kurt Waldner, "Transformer Insulation Life Assessment", IEEE Transaction on Power Delivery, Vol.21, No.1,Jan2006.

[2] M. K. Pradhan, "Assessment of the Status of Insulation during Thermal Stress Accelerated Experiments on Transformer Prototypes", IEEE Transactions on Dielectrics and Electrical Insulation Vol. 13, No. 1; February 2006, pp. 227-237.

[3] Jouni K. Pylvänäinen, Kirsi Nousiainen, and Pekka Verho, "Studies to Utilize Loading Guides and ANN for OilImmersed Distribution Transformer Condition Monitoring”, IEEE Transaction on Power Delivery, Vol. 22, No. 1, January 2007, pp.201-207.

[4] S. M. Strachan, S. Rudd, S. D. J. McArthur, M. D. Judd, S. Meijer and E. Gulski, "Knowledge-Based Diagnosis of Partial Discharges in Power Transformers", IEEE Transactions on Dielectrics and Electrical Insulation Vol. 15, No. 1; February 2008,pp.259-268.

[5] A. B. Lobo Ribeiro, N. F. Eira, J. M. Sousa, P. T. Guerreiro, and J. R. Salcedo, "Multipoint Fiber-Optic HotSpot Sensing Network Integrated Into High Power Transformer for Continuous Monitoring", IEEE Sensors Journal, Vol.8 no.7, July 2008, pp. 1264 - 1267. 


\section{International Journal of Science and Research (IJSR) \\ ISSN (Online): 2319-7064 \\ Index Copernicus Value (2013): 6.14 | Impact Factor (2014): 5.611}

[6] A. Shintemirov, W. H. Tang, and Q. H. Wu, "Transformer Core Parameter Identification Using Frequency Response Analysis", IEEE Transactions on Magnetics, Vol. 46, No. 1; January 2010, pp. $141-149$.

[7] A. Shintemirov, W.H. Tang and Q.H. Wu, "Transformer winding condition assessment using frequency response analysis and evidential reasoning", IET Electric Power Applications, 2010, Vol. 4, Issue 3, pp. 198-212.

[8] Victoria M. Catterson, Stephen D. J. McArthur, and Graham Moss, "Online Conditional Anomaly Detection in Multivariate Data for Transformer Monitoring", IEEE Transaction on Power Delivery, Vol. 25, No. 4, October 2010, pp. 2556 - 2564.

[9] M. Wang and A. J. Vandermaar "Review of condition assessment of power transformers in service", in IEEE Electrical insulation Magazine, vol. 18, no. 6, pp. 12-25, November/December 2002.

[10] David Woodcock "Risk-Based Reinvestment-Trends in Upgrading the Aged T\&D System", Energy Pulse, March 12, 2004.

[11] Saha T.K. Purkait P., Understanding the impacts of moisture and thermal ageing on transformer's insulation by dielectric response and molecular weight measurements, IEEE Transactions on Dielectrics and Electrical Insulation April 2008, Volume: 15 , Issue: 2 pp: $568-582$

[12] Ragini Agarwal, S V Kulkarni, B K Sahoo, V R Sardeshpande, Raghavendra Deshpande, Online Monitoring of Transformer: A case study.

[13]Zhiming Wang, Weichun Ge, Chenggang Wang, Chaoming Zeng, The Applications of Networking of Consumption Data Acquisition System by Combining Broadband Power line Communication and Wireless Communication , Innovative Smart Grid Technologies Asia (ISGT Asia), April 2012 IEEE.

[14] Dharmawan, H.A., Ali, S.A.M. , A Compact Remote Monitoring System for a Three-Phase 10-kVA EnergyEfficient Switchable Distribution Transformer, IEEE Transactions on Instrumentation and Measurement March 2012, Volume: 61, Issue: 3, pp: $618-628$

[15]Bing Li, Songyuan Li, Ling Chen, XushengLi,ShangyongQu, Design and realization of wireless remote image monitoring system based on GSM/GPRS , Third International Forum on Strategic Technologies, 2008. IFOST 2008, pp: 260 - 263

\section{Author Profile}

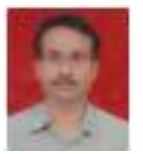

Bhupesh Nemichand Choudhari was born in Aurangabad, India. He received his B.E and M.E. in Electrical Engineering from Marathwada University, Aurangabad, India, in 1993 and 2000, respectively. He is currently an associate professor in the Department of Electrical Engineering, P.E.S. College of Engineering, Aurangabad, India. His current research interests include condition monitoring and fault diagnosis of electrical machines.

Makarand Sudhakar Ballal was born in Nagpur, India. He received his B.E. in Electrical Engineering from Marathwada University, Aurangabad, India in 1993. He received his M. Tech. and Ph.D. in Electrical Engineering

Volume 5 Issue 3, March 2016 www.ijsr.net

Paper ID: NOV161640 